\title{
Ultraschall der Achsel verbessert das Staging bei Brustkrebs
}

\begin{abstract}
Patientinnen mit triple-negativem Mammakarzinom (TNBC) erhalten in der Regel eine neoadjuvante Chemotherapie. Reichen körperliche Untersuchung und Mammografie aus, um befallene axilläre Lymphknoten vor der Therapie sicher zu erkennen und für die spätere Operation zu markieren?
\end{abstract}

\begin{abstract}
etrospektiv werteten Forscher diagnostische bzw. Staging-Maßnahmen vor Beginn der Therapie (körperliche Untersuchung, Mammografie, Ultraschall von Brust und regionalen Lymphknoten und ggfs. eine Biopsie verdächtiger Lymphknoten) und die definitive lokoregionäre Therapie von Patientinnen aus, bei denen am M. D. Anderson Cancer Center zwischen 2004 und 2011 ein TNBC behandelt worden war. Dabei verglichen sie besonders den Effekt einer Ultraschalluntersuchung regionaler Lymphknoten auf die Therapiestrategie.

Bei 111 (19,4\%) der 572 Patientinnen mit TNBC führte der regionale Ultraschall mit ggfs. folgender Feinnadelaspiration zum Upstaging gegenüber körper-
\end{abstract}

licher Untersuchung und Mammografie allein. In 50 Fällen (8,7\%) wurde eine bis dahin als nodal-negativ eingestufte Erkrankung nodal-positiv, bei 47 Patientinnen $(8,2 \%)$ änderte sich das Staging von cN1 zu cN3, bei 12 (2,1\%) von cN2 zu cN3 und bei $2(0,4 \%)$ von $\mathrm{cN} 3 \mathrm{zu}$ einem noch höheren Nodalstatus.

Andererseits ergab bei 28 Patientinnen mit tastbaren Lymphknoten bzw. in 1 Fall einer unklaren axillären Adenopathie die Sonografie einen nodal-negativen Status. Das Upstaging bei 19,2\% der Patientinnen führte im Vergleich $\mathrm{zu}$ Frauen ohne solche Neubewertung zu mehr neoadjuvanten Chemotherapien (91,9 vs. 51,2\%; $\mathrm{p}<0,0001)$ und einer häufigeren axillären Lymphadenektomie
(99,1 vs. $34,5 \%$; $\mathrm{p}<0,0001)$ bzw. Bestrahlung des regionalen Lymphknotengebiets (88,2 vs. $29,1 \% ; \mathrm{p}<0,0001)$.

Fazit: Bei jeder 5. Patientin wird durch die Ultraschalluntersuchung der regionalen Lymphknoten eine nodale Erkrankung aufgedeckt, was zum Upstaging mit unmittelbarer Auswirkung auf die Therapieplanung führt. Das stützt die Empfehlung des National Comprehensive Cancer Network, bei nodal-negativem TNBC vor der neoadjuvanten Chemotherapie immer eine Sonografie der regionalen Lymphknoten vorzunehmen und bioptisch gesicherte Lymphknotenmetastasen zu klippen, um eine nodale Resterkrankung später sicher entfernen zu können. Friederike Klein

Shaitelman SF et al. Role of Ultrasonography of Regional Nodal Basins in Staging Triple-Negative Breast Cancer and Implications For Local-Regional Treatment. Int J Radiat Oncol Biol Phys. 2015;93(1):102-10.

\section{Mammakarzinom mit niedrigem Rezidivrisiko: Verzicht auf adjuvante Chemotherapie möglich}

\author{
Einem Teil der Patientinnen mit östrogenrezeptorpositivem Brustkrebs \\ könnte möglicherweise eine adjuvante Chemotherapie erspart werden, \\ wie Studiendaten zeigen.
}

$B^{\mathrm{e}}$ ei Patientinnen mit einem östrogenrezeptorpositiven Mammakarzinom orientiert sich die Empfehlung für oder gegen eine adjuvante Chemotherapie derzeit an den klinisch-pathologischen Eigenschaften des Tumors. Doch einige Patientinnen sind damit möglicherweise übertherapiert, eine alleinige endokrine Therapie könnte für sie ausreichend sein. Aus früheren Studien weiß man, dass Genexpressionsanalysen zusätzliche Informationen bezüglich der Prognose liefern, unabhängig von den klinisch-pathologischen Eigenschaften des Tumors. Sie geben auch Hinweise auf den Nutzen einer adjuvanten Chemotherapie. Um Daten mit höherer Aussagekraft zu erhalten, wurde hierzu nun eine prospektive Studie durchgeführt, in die 10.253 Frauen mit hormonrezeptorpositivem, HER2-negativem Mammakarzinom $(1,1-5,0 \mathrm{~cm}$ in der größten Ausdehnung oder $0,6-1,0 \mathrm{~cm}$ und intermediäres oder hohes Tumorgrading) ohne Lymphknotenbefall rekrutiert wurden. Alle mussten die Kriterien für eine adjuvante Chemotherapie (auf Basis klinisch-pathologischer Eigenschaften) erfüllen. Die Gewebeproben wurden einer Expressionsanalyse von $21 \mathrm{Ge}$ nen (Oncotype DX) unterzogen, die einen Score für das Rezidivrisiko ergab. 1.626 Frauen mit sehr niedrigem Rezidivrisiko (Score 0-10 auf einer Skala von 0-100) erhielten nur eine endokrine Behandlung ohne Chemotherapie.
Nach 5 Jahren waren 93,8\% ohne eine invasive Erkrankung am Leben. Darüber hinaus waren 99,3\% der Patientinnen frei von Fernmetastasen und 98,7\% hatten weder Fernmetastasen noch lokoregionäre Rezidive. Die Rate für das $\mathrm{Ge}$ samtüberleben betrug 98,0\%.

Fazit: Bei einigen Patientinnen mit hormonrezeptorpositivem, HER2-negativem Mammakarzinom ohne Lymphknotenbefall kann man möglicherweise auf eine adjuvante Chemotherapie verzichten, selbst wenn sie auf der Basis klinisch-pathologischer Tumoreigenschaften die Kriterien für eine adjuvante Chemotherapie erfüllen. Voraussetzung ist, dass sie ein günstiges Genexpressionsprofil aufweisen. In der vorliegenden Studie blieben die Rezidivraten bei diesen Patientinnen sehr niedrig, auch wenn sie lediglich eine endokrine Therapie erhielten. Judith Neumaier

Sparano JA et al. Prospective Validation of a 21Gene Expression Assay in Breast Cancer. N Engl J Med. 2015;373(21):2005-14. 